\title{
Walter Stark (1924-2009)
}

Am 20. Februar 2009 vollendete sich das Leben von Prof. Dr. Walter Paul Ernst Stark nach längerer Erkrankung. Walter Stark wurde am 24. Juli 1924 in Danzig-Lagefuhr geboren. Nach frohen Kindertagen in einem Dorf an der Nogat, wo sein Vater Dienst als Grenzbeamter zu Polen tat, erlebte er den Beginn des zweiten Weltkrieges mit dem Beschuss der Westernplatte. Nach dem Abitur wurde er nach kurzer Arbeitsdienstzeit zur Wehrmacht gezogen. Aus englischer Kriegsgefangenschaft kommend ging er in die Nähe von Greifswald zu seiner Mutter und seinem älteren Bruder. Er begann ein Lehrerstudium in Greifswald im Jahr 1946 und konnte dieses für die Fächer Geschichte und Latein 1951 abschließen. Bis 1962 war er Lehrer an der Arbeiter- und Bauernfakultät der Universität Greifswald. Nach deren Schließung bot sich ihm die Möglichkeit, am Historischen Institut als wissenschaftlicher Mitarbeiter zu arbeiten und sich zu qualifizieren. 1982 wurde Walter Stark Hochschuldozent und 1986 dann Professor. In der Lehre hat er zu seinem Leidwesen keine Hansegeschichte lehren können. Er las zur Alten Geschichte, zur Geschichte Preußens und Deutschlands im 19. und beginnenden 20. Jahrhundert. In seinen Seminaren lernten die Studenten die wissenschaftliche Arbeit eines Historikers mit striktem Quellenbezug und dem Blick auf das Ganze. Sein Universalwissen auf dem Gebiet der Geschichte wie auch der deutschen Literatur zog viele in seinen Bann. Er vermittelte das geistige Band geschichtlicher Zusammenhänge, das für das Urteil des Historikers grundlegend ist. Seine Lehre zeichnete sich durch Exaktheit und Anschaulichkeit im Vortrag aus, der durch eingestreute Anekdoten und Histörchen gewürzt wurde. Jene wurden zugleich amüsante Gedächtnisstuitzen und waren aufs äußerste verknappte Charakteristika wesentlicher Erscheinungen.

Walter Stark wurde im Jahr 1969 mit einer Arbeit zum Verhältnis seiner Heimat- und Hansestadt Danzig zum Hansehaupt Lübeck promoviert. Danzig als Motor des preußischen Widerstands gegen die Herrschaft des Deutschen Ordens und als Befürworter der Herrschaft des 
polnischen Königs zeigte sich in der zweiten Hälfte des 15. Jahrhunderts selbstbewusst in seinem eigenständigen Handel in der Hanse. Durch eine umfassende Analyse des gedruckten und umfangreichen ungedruckten Quellenmaterials gelang Walter Stark der ïberzeugende Nachweis, dass der vieldiskutierte ,innerhansische Separatismus“ nicht nur durch psychologisierende Betrachtung oder durch die Betonung irgendwie gearteter ,besonderer Traditionen“ erklärt werden kann, sondern dass er vielmehr Ausdruck handelspolitischer Gegensätze innerhalb der Hanse war. die aus unterschiedlichen Entwicklungen einzelner Städte bzw. ganzer Städtegruppen resultierten. Diese Arbeit fand wegen ihres umfangreichen Quellenbezugs große Anerkennung.

Er war nunmehr anerkanntes Mitglied in der Greifswalder Arbeitsgruppe zur Hanseforschung unter Leitung von Johannes Schildhauer geworden. Die aufwendigen Archivstudien begründeten wissenschaftliche Kontakte zu polnischen Forscher in Gdansk, Warzsawa, Torun und Szczecin, die in folgenden Jahren ausgebaut wurden. Die in der Kinderzeit erworbenen Polnischkenntnisse waren dabei immer sehr hilfreich.

Gemeinsam mit Johannes Schildhauer und Konrad Fritze verfasste er die einzige in der DDR-Zeit geschriebene marxistische Hansegeschichte, die nicht nur in der DDR in sieben Auflagen, sondern auch in der Bundesrepublik Verbreitung und Beachtung fand.

Einen großen Beitrag zur hansischen Wirtschaftsgeschichte leistete Walter Stark durch seine Arbeiten zum hansischen Handel und zum Handelsprofit des hansischen Kaufmanns. Seine Habilitations-Schrift zum hansischen Handelsprofit auf der Grundlagen vor allem des Nachlasses von Hildebrand Veckinchusen und des Danziger Kaufmanns Johann Pisz brachte im die ungeteilte Anerkennung der Hanseforscher wie auch darüber hinaus. In subtiler und scharfsinniger Analyse des Quellenmaterials konnte er nachweisen, dass der durchschnittliche Handelsprofit im doppelten bis dreifachen des geltenden Rentenzinses gelegen hat. Die Art und Weise der Berechnung durch die Beachtung des Hin- und Rückkaufes als einem Geschäftsvorgang machte dieses Ergebnis möglich.

Das Streben nach möglichst hohen und gesicherten Handelsprofiten war die wichtigste Triebfeder für alle Aktionen der mittelalterlichen Kaufleute und demzufolge auch direkt oder indirekt mitbestimmend für die von ihnen ausgehenden Wirkungen auf die Entwicklung der sozialen und politischen Verhältnisse sowie der Kultur. Walter Stark gelang hier erstmalig, die Frage nach Entstehung und Entwicklung der hansischen Handelsprofite exakt zu beantworten, indem er die Methode zur Lösung dieser Problematik fand und überzeugend beschrieb. 
Zum Werk Walter Starks gehören auch jene Arbeiten, in denen er Teilaspekte hansischer Geschichte aufgriff. Hier sind einige Arbeiten zum Salzhandel in der Hanse ebenso zu nennen wie jene zur Handelspraxis des hansischen Kaufmanns oder der Artikel zum Utrechter Frieden.

Nach dem ersten Band der von Michail Lesnikov herausgegebenen Handlungsbücher des Hildebrand Veckinchusen lag auch die Druckvorlage des zweiten Bandes Ende der siebziger Jahre vor, die aber hinausgezögert wurde, da die Kritik am ersten Band es geraten sein ließ, noch einmal eine Kollationierung der Vorlage mit dem Original vorzunehmen. 1983 war der M. Lesnikov in Moskau verstorben. Die Hansische Arbeitsgemeinschaft in der DDR suchte einen Bearbeiter und fand inn in Walter Stark. Er wohnte in Berlin Ost und fuhr täglich nach Berlin West ins Geheime Staatsarchiv preußischer Kulturbesitz in Berlin-Dahlem, wo er die später nach Tallinn zurückgegebenen Handlungsbücher einsehen konnte. Diese Arbeit war 1988 abgeschlossen. Der Verlag sah aber keine Möglichkeit, das vorliegende Manuskript setzen zu lassen. So setzte sich Walter Stark hin und begann mit dem Abtippen des Manuskriptes. Darüber ging die Wende hinweg und das Projekt kam ins Stocken. Der Vorstand des Hansischen Geschichtsvereins nahm sich dieses einst selbst initiierten Projektes wieder an. Über knapp zwei Jahre digitalisierte Herr Dr. Marc Löwener, Berlin, in Abstimmung mit Walter Stark die Handlungsbücher, was die umgehende Korrektur der Eingabe mit einschloss. Krankheiten des Bearbeiters unterbrachen diese Arbeit immer wieder. Der Wunsch. diese Arbeit noch zum glücklichen Ende bringen zu können, stärkte seinen Genesungswillen bis zuletzt, jedoch es war ihm dies versagt.

Walter Stark gehörte jener Generation von DDR-Historikern an, die noch bei einem bürgerlichen Historiker, Adolf Hofmeister, das solide Handwerk eines Historikers erlernt hatten und die marxistische Methode und Lehre einerseits aus Lebenserfahrung, andererseits aus erkenntnistheoretischer Sicht nutzten. Seine wissenschaftliche Arbeiten sind quellengestützt und -gesteuert und haben dadurch Bestand und Anerkennung gefunden.

Die Hanseforschung verliert mit ihm einen verdienstvollen und profilierten Forscher. Es bleibt darüber hinaus die Erinnerung an einen streitbaren. unbestechlichen und hochachtbaren Menschen.

Horst Wernicke, Greifswald 